\title{
Neuroendoscopic biopsy of brain lesions: accuracy and complications
}

\author{
Alexandre Varella Giannetti, MD, PhD, ${ }^{1,2}$ Alexandre Yugo Holayama Alvarenga, MD, ${ }^{2}$ \\ Thiago Oliveira Lemos de Lima, ${ }^{1}$ Hugo Abi-Sáber Rodrigues Pedrosa, MD, ${ }^{1}$ \\ and Mark M. Souweidane, MD ${ }^{3}$
}

\begin{abstract}
${ }^{1}$ Department of Surgery, School of Medicine, and 'Service of Neurosurgery, Hospital das Clínicas, Universidade Federal de Minas Gerais, Belo Horizonte, Brazil; and ${ }^{3}$ Department of Neurological Surgery, Weill Cornell Medical College, New York, New York
\end{abstract}

OBJECT The authors conducted a study to analyze the accuracy of neuroendoscopic biopsies of ventricular and periMETHODS A total of 50 patients with different diseases consecutively underwent endoscopic biopsy procedures. The biopsy result was considered diagnostic if the pathologist reported any specific finding on which clinicians could base decisions about treatment or observation. The biopsy result was referred to as accurate if the results matched results of a sample obtained later or if the treatment response and disease evolution were compatible with the diagnosis. The biopsy result was considered inaccurate if results showed any relevant differences from those of the later sample or if the patient's disease did not evolve as expected. Complications were recorded and compared with those found in a literature review.

RESULTS For 2 patients, the procedure had to be terminated. The biopsy diagnostic rate was $89.6 \%$, and the accuracy rate was $86 \%$. Complications associated with the procedure were 3 hemorrhages (6\%), 2 infections (4\%), and 1 death (2\%); no reoperations were needed.

CONCLUSIONS Endoscopic biopsy seems to be an accurate procedure with acceptable morbidity and mortality rates. http://thejns.org/doi/abs/10.3171/2014.9.JNS132648

KEY WORDS neuroendoscopy; brain tumor; endoscopic biopsy; intraventricular lesion; oncology

A $\mathrm{s}$ a result of recent improvements in lens technologies and optics, the use of neuroendoscopic procedures has mushroomed. The utility of neuroendoscopic biopsy for obtaining brain tissue samples has been reported on; ${ }^{1-3,5-8,10-16,18-23}$ however, despite the substantial number of articles addressing this issue, the accuracy of this technique has not been totally clarified. Many articles do not compare the endoscopic diagnosis with diagnoses made by any other method or with the established treatment. In addition, almost all papers in the literature consider tumor cases only. The complication rate is variable because of differences in techniques and the small number of cases assessed in most published studies. Our objective was to analyze the accuracy of neuroendoscopic biopsy results by comparing them with results of biopsies performed later and thereby evaluating the treatment evolution. The difficulties and complications encountered are presented and compared with those reported in the literature.

\section{Methods}

From January 2002 through March 2013, a total of 407 purely neuroendoscopic procedures were performed by the same neurosurgeon (A.V.G.) at the Service of Neurosurgery of Hospital das Clínicas of Federal University of Minas Gerais, Brazil. From a database of these procedures, we selected 50 patients with intraventricular or periventricular lesions from which biopsy samples had been obtained. The inclusion criteria were as follows: patients of all ages; any pathology inside or at the walls of the supratentorial ventricular system; and intent for a biopsy, regardless of its success. Patients who underwent tumor resection (such as for colloid cysts), cyst aspiration,

ABBREVIATION EVD = external ventricular drain.

SUBMITTED November 30, 2013. ACCEPTED September 23, 2014.

INCLUDE WHEN CITING Published online October 31, 2014; DOI: 10.3171/2014.9.JNS132648.

DISCLOSURE This work had the support of Pró-Reitoria de Pesquisa of the Universidade Federal de Minas Gerais. 
or cyst removal (cysticercosis) were not included in the study.

After the research project was approved by the Ethics Committee of the Federal University of Minas Gerais, the patients' charts and histopathology reports were reviewed and patients were contacted for follow-up. The data were transcribed onto a protocol form designed specifically for this research and then transferred to an Excel spreadsheet.

Of the 50 patients, 27 were female and 23 were male. Ages ranged from 5 months through 68 years; 23 were children younger than 17 years, and 27 were adults.

Hydrocephalus was present in all but 2 patients. A CSF diversion procedure was conducted simultaneously with the biopsy procedure by a variety of approaches, depending on the site of CSF flow obstruction. In general, an endoscopic third ventriculostomy was performed when the pathology was in the posterior region of the third ventricle; this step was always performed before the biopsy. For patients in whom the lesion occupied the anterior portion of the third ventricle or the region of the foramen of Monro, an endoscopic septostomy was performed and a ventriculoperitoneal shunt was implanted. For these patients, the surgical procedure was initiated by opening the abdomen, passing the distal catheter under the subcutaneous layer between this incision and the frontal access, and then completing the endoscopic step. The last step, all performed under endoscopic visualization, comprised the biopsy, subsequent fenestration of the septum pellucidum, and insertion of the ventricular catheter. For cases in which the area of the pathological process responsible for the obstruction of the foramen of Monro was small, the biopsy itself was sufficient to release the CSF. To avoid restenosis, a stent (catheter) could be implanted to maintain communication between the lateral and third ventricles. A septostomy was added in these situations. An external ventricular drain (EVD) was inserted in cases of infection or significant intraoperative bleeding.

All endoscopic procedures were performed with a DECQ endoscope of $3.5 \times 5.2-\mathrm{mm}$ outer diameter and $0^{\circ}$ rigid-rod lens (KARL STORZ GmbH \& Co. KG). The biopsy forceps were $1.3 \mathrm{~mm}$ in diameter. After endotracheal induction of general anesthesia and prophylactic administration of antibiotics, patients were positioned with their head in a neutral position over a horseshoe head support. When a shunt insertion was planned, the patient's head was turned to the contralateral side. For all patients, the access was frontal, 1-2 cm anterior to the coronal suture and $3 \mathrm{~cm}$ off the midline. Access was almost always from the right side, whether required or elected. After recognition of the ventricular system, the lesion was approached, and at least 5 biopsy specimens were taken. An a priori cauterization of the lesion surface was never performed. Bleeding was controlled with warm Ringer solution. For large lesions, biopsy specimens were taken from the surface and from the interior of the lesion. Intraoperative frozen analysis was not performed.

The tissue samples were embedded in paraffin. All were processed for $\mathrm{H} \& \mathrm{E}$ staining, and some were followed by immunohistochemical staining when indicated.

The treatment plan was based on the histopathological diagnosis and usually consisted of 1 or more of the fol- lowing: open surgery (especially for tumors, depending on their natural history), radiotherapy, chemotherapy, and specific medical treatment for infectious diseases. In cases of benign and unresectable neoplasms or ependymitis secondary to cysticercosis, no treatment was instituted. Patients were followed up on an ambulatory basis.

To confirm the diagnosis made from the biopsy specimen and to verify its accuracy, the histopathological results were compared with the following: 1) new specimens taken after an open surgery; 2) the response to medical treatment, chemotherapy, and/or radiotherapy; and 3) the clinical and radiographic outcomes for patients managed without treatment. Three patients died soon after the endoscopic procedure; 1 death was associated with a hemorrhagic complication and 2 were associated with previous poor overall health of the patient. With regard to the latter 2 patients, in 1, the lesion bled spontaneously and the patient was comatose when she arrived at the hospital; in the other, a systemic infection related to immunosuppression evolved into sepsis a few weeks after the surgery. In addition to these 3 patients, 2 patients who were lost to follow-up and 2 others who did not undergo a biopsy were excluded from the final analysis for accuracy. The endoscopic biopsy was considered accurate when it met the following criteria: 1) histopathological diagnosis matched that of the sample obtained during craniotomy; 2 ) the response to the treatment was consistent with the diagnosis; and 3) the follow-up symptoms and images were compatible with the diagnosis. The endoscopic biopsy was considered inaccurate when any of the following were true: 1) the new sample obtained during craniotomy suggested a new diagnosis or substantial change in tumor grade (thereby indicating changes that could affect treatment or prognosis); or 2) the response to treatment or evolution among those followed up without treatment differed from the expected response.

Only threatening complications associated with the endoscopic procedures were analyzed. Minor complications and transitory deficits were not reported because they might be underestimated in this type of retrospective study. Because hydrocephalus treatment was out of the scope of this study, information about shunt dysfunction was not collected. Hemorrhagic complication was defined as an important bleeding that led to the termination of the procedure, that required the unplanned insertion of an EVD, or that was detected on the postoperative scan and required further surgery. These complications could be associated with the biopsy itself, to the opening of the third ventricle, or to the endoscopic navigation. All other hemorrhages were disregarded. The diagnosis of bacterial meningitis or ventriculitis was defined by CSF analysis and positive culture. Reoperation was defined as any second surgery necessary to overcome a complication of endoscopy. EVD insertion during the endoscopic procedure was not considered a reoperation, even if it had not been previously scheduled.

\section{Results}

Of the 50 patients who underwent endoscopic biopsies for intraventricular or periventricular lesions, the proce- 
dure was terminated for 2 patients. In the first patient, bleeding occurred during the third ventriculostomy, and the biopsy of a pineal region tumor was terminated. In the second patient, a small lesion inside the aqueduct was not easy to approach; considering that the lesion could be benign and understanding the risk of mesencephalic injury, the surgeon aborted the biopsy attempt. In total, 48 biopsies (96\%) were performed.

A total of 48 patients had symptomatic hydrocephalus, which was managed by at least 1 of the following neuroendoscopic procedures: third ventriculostomy (29 patients [60\%]), septostomy (22 [46\%]), opening of the foramen of Monro (3 [6\%]), aqueductoplasty (1 [2\%]) and communication of a trapped temporal horn to the ambient cistern (1 [2\%]). A ventriculoperitoneal shunt was inserted in 10 patients $(21 \%)$, a stent was implanted in 3 patients (6\%), and an EVD was left in 4 patients (8\%).

Table 1 shows the diagnostic results obtained from the 48 successfully biopsies. Treatment planning was based on these findings, except for the 3 patients who died soon after the surgical procedure. Of the remaining 45 patients, $14(31 \%)$ underwent a craniotomy, with or without complementary therapy; 8 (18\%) received chemotherapy and/or radiotherapy; 4 (9\%) received treatment for a specific infectious disease (2 cryptococcosis, 1 toxoplasmosis, and 1 paracoccidioidomycosis); and 19 (42\%) did not receive any new treatment. Two patients were lost to follow-up.

Table 2 shows the accuracy of the endoscopic biopsy. Of the remaining 43 patients, biopsy results were accurate for $37(86 \%)$, according to our protocol. For 4 patients, the new histopathological finding changed the previous diagnosis. The lesion with a biopsy result of normal tissue turned out to be a Grade IV glioma. The pineal region lesion with inconclusive biopsy result was a teratoma. For 2

TABLE 1. Histopathological diagnoses for 48 successful neuroendoscopic biopsies

\begin{tabular}{lcc}
\hline \multicolumn{1}{c}{ Diagnosis } & No. & $\%$ \\
\hline Glioma Grade II & 9 & 18.7 \\
\hline Pilocytic astrocytoma & 8 & 16.7 \\
\hline Germinoma & 5 & 10.4 \\
\hline Cysticercosis & 4 & 8.3 \\
\hline Pineoblastoma & 3 & 6.2 \\
\hline High-grade glioma (III \& IV) & 2 & 4.2 \\
\hline Cryptococcosis & 2 & 4.2 \\
\hline Round cell tumor & 2 & 4.2 \\
\hline Medulloblastoma (metastasis) & 1 & 2.1 \\
\hline Hamartoma & 1 & 2.1 \\
\hline Craniopharyngioma & 1 & 2.1 \\
\hline Pineocytoma & 1 & 2.1 \\
\hline Epidermoid tumor & 1 & 2.1 \\
\hline Leukoencephalopathy w/ cysts \& calcifications & 1 & 2.1 \\
\hline Toxoplasmosis & 1 & 2.1 \\
\hline Paracoccidioidomycosis & 1 & 2.1 \\
\hline Nonspecific & 4 & 8.3 \\
\hline Normal tissue & 1 & 2.1 \\
\hline
\end{tabular}

TABLE 2. Accuracy of neuroendoscopic biopsy results

\begin{tabular}{ll}
\hline \multicolumn{1}{c}{ Biopsy Result } & No. $(\%)$ \\
\hline Accurate & \\
\hline Diagnosis confirmed by new sample & 10 \\
\hline Diagnosis compatible w/ treatment response & 11 \\
\hline Diagnosis compatible w/ follow-up & 16 \\
\hline Total accurate & $37(86)$ \\
\hline Inaccurate & \\
\hline New biopsy result differed & 4 \\
\hline Clinical course not compatible w/ diagnosis & 1 \\
\hline Inconclusive finding & 1 \\
\hline Total inaccurate & $6(14)$ \\
\hline Total included & 43 \\
\hline Excluded & 3 \\
\hline Death & 2 \\
\hline Lost to follow-up & 2 \\
\hline Biopsy not performed & 7 \\
\hline Total excluded & $50(100)$ \\
\hline Total biopsies & \\
\hline
\end{tabular}

patients, the lesion grade changed; what biopsy indicated as a high-grade glial neoplasm was actually a teratoid rhabdoid tumor, and what biopsy indicated as a pineocytoma was actually a pineoblastoma. For 1 patient, the clinical course was incompatible with the diagnosis. This patient had a diffuse tumor, the biopsy of which showed a Grade II glioma. However, the tumor progressed rapidly, resembling a high-grade lesion. One patient had acquired immunodeficiency syndrome and a mesencephalic lesion; he underwent a third ventriculostomy and a stereotactically guided endoscopic biopsy. The histological result was inconclusive. No new treatment or biopsy was performed. This biopsy was also considered inaccurate.

Hemorrhagic complications of the neuroendoscopic procedures occurred in 3 patients (6\%); in 2 patients, an EVD was inserted, and a shunt was placed a few days later. There were 2 cases (4\%) of ventriculitis, in both of which an EVD was used and antibiotics were administered without major consequences. There was 1 death associated with the neuroendoscopic procedure. This patient experienced an significant intraoperative bleed, and despite placement of an EVD and a definitive shunt, she died on the 6th postoperative day.

\section{Discussion}

The medical literature includes several articles addressing the usefulness of endoscopic biopsy for the management of intraventricular and periventricular lesions. The diagnostic yield of this type of procedure varies between $69.6 \%$ and $100 \%{ }^{1,2,4-12,15,17-21}$ This wide variation may be explained by several methodological differences. Some articles consider only tumors, ${ }^{1,2,5,9-11,15,18}$ others assess only a single, specific site (such as the pineal region) $2,10,13,18$ or limit patients to a selected age range (e.g., children). ${ }^{1,5,19,20}$ Other possible explanations for the differences in success 
rates include variation in the type of endoscope (rigid or flexible), ${ }^{7}$ in the size of the tumor forceps cups, ${ }^{1,7}$ in the number of samples obtained, ${ }^{7}$ and in the experience of the surgeon..$^{1,4,10,20}$ However, in our opinion, the most influential factor is associated with the authors' definition of accuracy. Some authors did not include in their final analysis patients for whom successful biopsies were not achieved. ${ }^{10}$ Others considered any histopathological abnormalities to be diagnostic., ${ }^{2,17,21}$ According to this last definition, our rate of positive results would be $97.9 \%$; the corresponding value in the literature ranges between $82.6 \%$ and $100 \%$ (Table 2). ${ }^{1,2,4-12,15,17-21}$

However, if a biopsy is performed to establish a possible treatment, we cannot count a nonspecific histopathological result as positive. Depreitere et al. classified the pathological report into 4 categories: Level I, fully diagnostic with no reservations; Level II, diagnostic with some reservations from the pathologist but with strong arguments for the preferred diagnosis; Level III, definitely reflective of abnormal or neoplastic tissues but problematic in coming to definitive diagnosis; and Level IV, not reflective of abnormal tissue or unable to make an interpretation. ${ }^{5}$ If we consider Levels I and II to be sufficient for the management of the patients, we (as do other authors) placed them into the same category as diagnostic biopsies. When this was done, our rate of positive results dropped to $89.6 \%$, whereas the rates of nonspecific and normal findings were $8.3 \%$ and $2.1 \%$, respectively. Our literature review retrieved rates of diagnostic, nonspecific, and normal findings of $65.4 \%-100.0 \%, 0 \%-34.6 \%$, and $0 \%-17.4 \%$, respectively (Table 3 ). ${ }^{1,2,4-12,15,17-21}$

Nevertheless, a positive diagnostic biopsy result does not necessarily mean that the diagnosis corresponds to the reality. To confirm the diagnosis, the findings from an endoscopic biopsy should ideally be compared with those of a larger sample. Many articles have cited a small number of cases showing the existence of mismatched results. Recently, Constantini et al. conducted a multicentric survey of 293 cases from different countries. Of these, 78 patients $(27 \%)$ whose biopsy results were considered informative underwent open surgery, and the 2 samples were compared; a complete match of diagnoses was found for $82 \%$ and mismatched but not meaningful (similar) results were found for $6.4 \%$. However, the mismatches were meaningful for $11.5 \%$ of the cases. ${ }^{4}$ Using the same method, we found a meaningful mismatch rate of $16.7 \%$ ( 2 of 12 cases). Our literature review found a total of 125 cases (including the 12 cases herein described) in which it was possible to compare the endoscopically biopsied sample to a new sample that had been obtained from open surgery. When we combined these data and analyzed them, we found complete match, similar, and mismatch rates of $82.4 \%, 7.2 \%$, and $10.4 \%$, respectively (Table 4). ${ }^{1,4,5,10,12,15}$

If this method of sample comparison seems very reliable from a scientific view point, it also presents some bias: 1) only the minority of patients who had undergone biopsy were enrolled; 2) all biopsied compared were from tumors; and yet 3) not all patients with tumors were included (only patients for whom an open approach would be considered a beneficial treatment were included). Still, according to
Constantini et al., in 4 cases of mismatch, the grade of the tumor turned out to be high. ${ }^{4}$ If we consider that some patients with benign tumors will not undergo surgery, there are probably patients in the excluded group who have received a misdiagnosis. To overcome these methodological issues, we conducted the study reported here. Our method enabled us to enroll all types of patients, including those without neoplastic diseases. However, we might perceive that not all of our patients will respond to treatment, even if the diagnosis is correct. The same reasoning is valid for those patients followed up without treatment, for whom the natural process of the disease differs from what is expected. Regardless, these methodological drawbacks, if present, would negatively influence our results. In other words, according to our method, the accuracy rate of endoscopic biopsies was at least $86 \%$.

Morbidity and mortality rates for patients undergoing endoscopic biopsy are low. For our retrospective analysis, we decided to not include minor complications because they would certainly be underestimated. In general, bleeding is the most important concern when one performs any type of endoscopic procedure. The definition of "important hemorrhage" might differ among authors; even we believe that this term is very subjective. Some articles have classified hemorrhagic complications as mild, moderate, and severe. ${ }^{4,12,17}$ Nevertheless, because almost all articles reviewed reported retrospective studies based on operative reports, we believe that their complication rates are possibly unreliable. In our study, we defined important bleeding as bleeding requiring the insertion of an EVD, leading to the termination of the procedure, or requiring a second surgery for adequate management. Among 50 procedures, $3(6 \%)$ hemorrhagic complications occurred and were associated directly with the biopsy procedure, with performing the third ventriculostomy, or with navigation with the endoscope. Using our definition of important bleed, we analyzed the data from the literature and recalculated the other authors' rates of hemorrhage. Rates of important bleeding varied from $0 \%$ to $9.7 \%$. Rates of infection, reoperation, and death varied from $0 \%$ to $6.5 \%, 0 \%$ to $6.5 \%$, and $0 \%$ to $6.3 \%$, respectively (Table 3)., 1,2,-12,15,17-21 However, because the number of patients in most series (including that reported herein) was low, any single complication might have considerably affected these rates. To overcome this effect, we collected data from all of the articles (including the series reported herein) and summed their absolute number of infections $(\mathrm{n}=18)$, reoperations $(\mathrm{n}=14)$, and deaths $(\mathrm{n}=8)$. We divided this sum by the number of surgeries (1615 cases) and arrived at percentage rates of $1.1 \%, 0.9 \%$, and $0.5 \%$, respectively (Table 3 ).

\section{Conclusions}

We analyzed the data obtained from 50 consecutively performed endoscopic biopsies for different diseases. Using a new method to calculate accuracy, we found that in at least $86 \%$ of the cases, the diagnostics provided by the biopsy results were accurate. We also compared our complication rates with those reported in the literature and concluded that this procedure is very safe. 


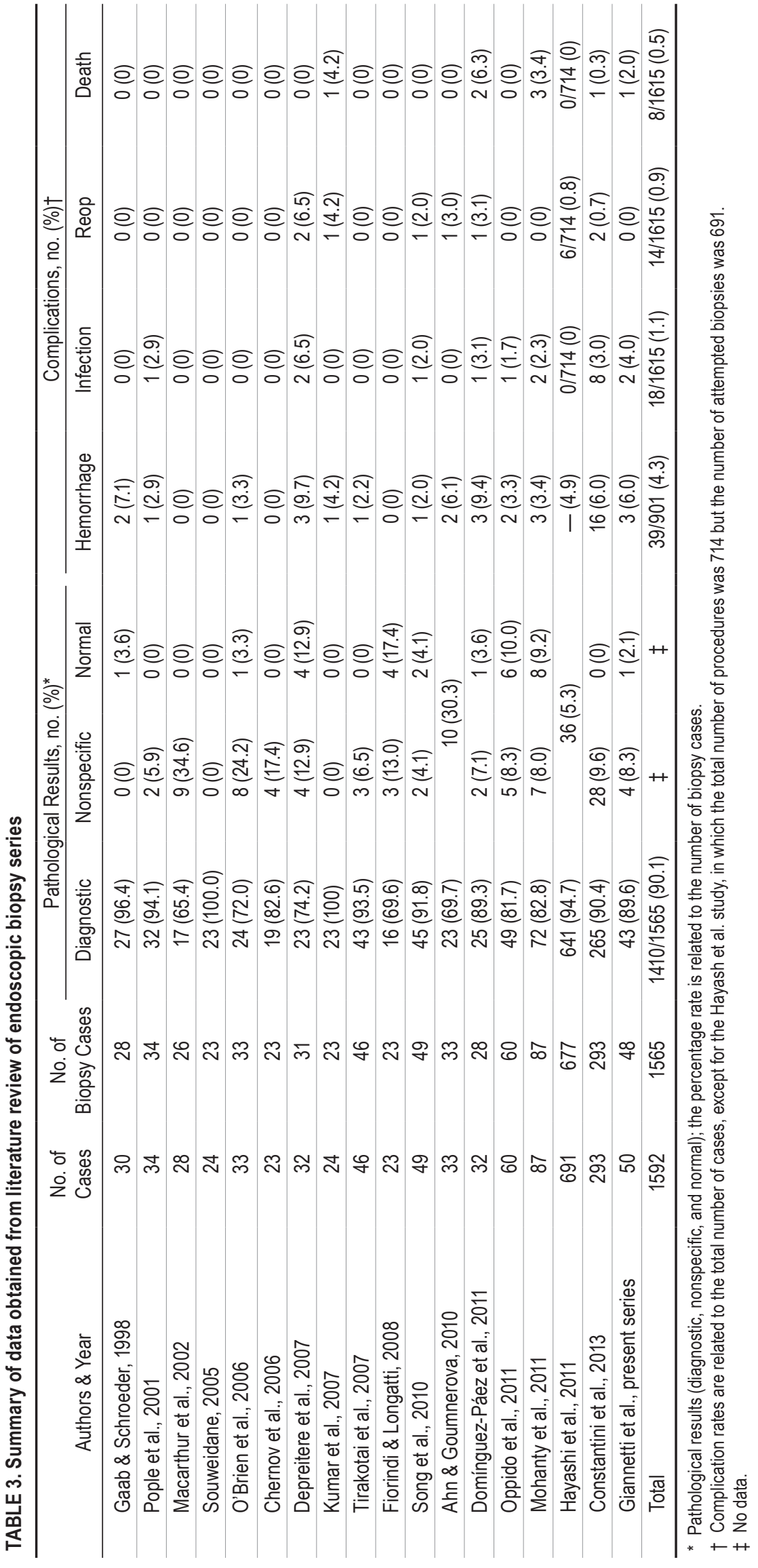


TABLE 4. Summary of data found in literature reporting comparisons of pathological findings of specimens taken by endoscopy and open surgery

\begin{tabular}{lcccc}
\hline & \multicolumn{4}{c}{ No. of Cases (\%) } \\
\cline { 2 - 5 } Authors \& Year & Overall & Matched & Similar & Mismatched \\
\hline O'Brien et al., 2006 & 5 & $4(80.0)$ & $0(0)$ & $1(20.0)$ \\
\hline Kumar et al., 2007 & 6 & $6(100)$ & $0(0)$ & $0(0)$ \\
\hline Depreitere et al., 2007 & 6 & $5(83.3)$ & $0(0)$ & $1(16.7)$ \\
\hline Ahn \& Goumnerova, 2010 & 2 & $2(100)$ & $0(0)$ & $0(0)$ \\
\hline Mohanty et al., 2011 & 16 & $12(75.0)$ & $4(25.0)$ & $0(0)$ \\
\hline Constantini et al., 2013 & 78 & $64(82.1)$ & $5(6.4)$ & $9(11.5)$ \\
\hline Giannetti et al., present series & 12 & $10(83.3)$ & $0(0)$ & $2(16.7)$ \\
\hline Total & 125 & $103(82.4)$ & $9(7.2)$ & $13(10.4)$ \\
\hline
\end{tabular}

\section{References}

1. Ahn ES, Goumnerova L: Endoscopic biopsy of brain tumors in children: diagnostic success and utility in guiding treatment strategies. Clinical article. J Neurosurg Pediatr 5:255-262, 2010

2. Chernov MF, Kamikawa S, Yamane F, Ishihara S, Kubo O, Hori T: Neurofiberscopic biopsy of tumors of the pineal region and posterior third ventricle: indications, technique, complications, and results. Neurosurgery 59:267-277, 2006

3. Chibbaro S, Di Rocco F, Makiese O, Reiss A, Poczos P, Mirone G, et al: Neuroendoscopic management of posterior third ventricle and pineal region tumors: technique, limitation, and possible complication avoidance. Neurosurg Rev 35:331340,2012

4. Constantini S, Mohanty A, Zymberg S, Cavalheiro S, Mallucci C, Hellwig D, et al: Safety and diagnostic accuracy of neuroendoscopic biopsies: an international multicenter study. Clinical article. J Neurosurg Pediatr 11:704-709, 2013

5. Depreitere B, Dasi N, Rutka J, Dirks P, Drake J: Endoscopic biopsy for intraventricular tumors in children. J Neurosurg (5 Suppl) 106:340-346, 2007

6. Domínguez-Páez M, Puch-Ramírez M, Rodríguez-Barceló S, Medina-Imbroda JM, Romero-Moreno L, Ibáñez-Botella G, et al: [Neuroendoscopic biopsy. Experience in 31 patients and literature review.] Neurocirurgia (Astur) 22:419-428, 2011 (Span)

7. Fiorindi A, Longatti P: A restricted neuroendoscopic approach for pathological diagnosis of intraventricular and paraventricular tumours. Acta Neurochir (Wien) 150:1235-1239, 2008

8. Gaab MR, Schroeder HWS: Neuroendoscopic approach to intraventricular lesions. J Neurosurg 88:496-505, 1998

9. Hayashi N, Murai H, Ishihara S, Kitamura T, Miki T, Miwa T, et al: Nationwide investigation of the current status of therapeutic neuroendoscopy for ventricular and paraventricular tumors in Japan. Clinical article. J Neurosurg 115:1147-1157, 2011

10. Kumar SVR, Mohanty A, Santosh V, Satish S, Devi BI, Praharaj SS, et al: Endoscopic options in management of posterior third ventricular tumors. Childs Nerv Syst 23:1135-1145, 2007

11. Macarthur DC, Buxton N, Punt J, Vloeberghs M, Robertson IJ: The role of neuroendoscopy in the management of brain tumours. Br J Neurosurg 16:465-470, 2002

12. Mohanty A, Santosh V, Devi BI, Satish S, Biswas A: Efficacy of simultaneous single-trajectory endoscopic tumor biopsy and endoscopic cerebrospinal fluid diversion procedures in intraand paraventricular tumors. Neurosurg Focus 30(4):E4, 2011

13. Morgenstern PF, Osbun N, Schwartz TH, Greenfield JP, Tsiouris AJ, Souweidane MM: Pineal region tumors: an optimal approach for simultaneous endoscopic third ventriculostomy and biopsy. Neurosurg Focus 30(4):E3, 2011
14. Najjar MW, Azzam NI, Baghdadi TS, Turkmani AH, Skaf G: Endoscopy in the management of intra-ventricular lesions: preliminary experience in the Middle East. Clin Neurol Neurosurg 112:17-22, 2010

15. O'Brien DF, Hayhurst C, Pizer B, Mallucci CL: Outcomes in patients undergoing single-trajectory endoscopic third ventriculostomy and endoscopic biopsy for midline tumors presenting with obstructive hydrocephalus. J Neurosurg (3 Suppl) 105:219-226, 2006

16. Oi S, Shibata M, Tominaga J, Honda Y, Shinoda M, Takei F, et al: Efficacy of neuroendoscopic procedures in minimally invasive preferential management of pineal region tumors: a prospective study. J Neurosurg 93:245-253, 2000

17. Oppido PA, Fiorindi A, Benvenuti L, Cattani F, Cipri S, Gangemi M, et al: Neuroendoscopic biopsy of ventricular tumors: a multicentric experience. Neurosurg Focus 30(4):E2, 2011

18. Pople IK, Athanasiou TC, Sandeman DR, Coakham HB: The role of endoscopic biopsy and third ventriculostomy in the management of pineal region tumours. Br J Neurosurg 15:305-311, 2001

19. Song JH, Kong DS, Shin HJ: Feasibility of neuroendoscopic biopsy of pediatric brain tumors. Childs Nerv Syst 26:15931598,2010

20. Souweidane MM: Endoscopic management of pediatric brain tumors. Neurosurg Focus 18(6A):E1, 2005

21. Tirakotai W, Hellwig D, Bertalanffy H, Riegel T: The role of neuroendoscopy in the management of solid or solid-cystic intra- and periventricular tumours. Childs Nerv Syst 23:653658,2007

22. Wong TT, Chen HH, Liang ML, Yen YS, Chang FC: Neuroendoscopy in the management of pineal tumors. Childs Nerv Syst 27:949-959, 2011

23. Yamini B, Refal D, Rubin CM, Frim DM: Initial endoscopic management of pineal region tumors and associated hydrocephalus: clinical series and literature review. J Neurosurg (5 Suppl Pediatrics) 100:437-441, 2004

\section{Author Contributions}

Conception and design: Giannetti. Acquisition of data: Giannetti, Alvarenga, de Lima, Pedrosa. Analysis and interpretation of data: Giannetti, de Lima, Souweidane. Drafting the article: Giannetti. Critically revising the article: Giannetti, Souweidane. Reviewed submitted version of manuscript: all authors. Approved the final version of the manuscript on behalf of all authors: Giannetti. Study supervision: Giannetti, Souweidane.

\section{Correspondence}

Alexandre Varella Giannetti, Rua Santa Catarina 1042, Apto 201, Belo Horizonte, MG, CEP: 30170-080, Brazil. email: agjg@terra. com.br. 